\title{
Perspective approach for cutaneous melanoma treatment by cationic peptides
}

\begin{abstract}
Background: Metastatic cutaneous melanoma is the most aggressive skin cancer with poor prognosis. As a rule, metastatic melanoma tumors with various localization have not complete recovery. Effective therapy of disseminated cutaneous melanoma remains an acute problem, in spite of the recent advances in immunotherapy and targeted molecular therapy. This paper describes a preliminary data obtained from the analysis of cytotoxicity and antitumor activity of some cationic peptides (CPs), in metastatic cutaneous melanoma cell lines generated from the biopsies and subcutaneous cutaneous melanoma xenografts in immunodeficient mice. Molecular mechanisms for CP selective cytotoxicity and specific antitumor effect are related with functional inhibition of chaperone proteins nucleolin and nucleophosmin followed by nucleolar stress
\end{abstract}

Objective: Evaluation of cytotoxicity and antitumor activities of some cationic peptides using in vitro and in vivo cell and mice xenograft models.

Material and methods: Three cutaneus melanoma cell lines generated from metastatic lymph nodes were used for in vitro analysis of cytotoxicity of 4 cationic peptides by MTT assay. Antitumor activity of the peptides was studied in vivo using subcutaneous xenografts generated from MelCher melanoma cell line. Flow cytometry and molecular docking were used for analysis of molecular mechanisms of cell death induced by peptides under study.

Results: The use of cationic peptides (CPs) with specific molecular structure for inducing selective tumor cell death, namely cutaneous melanoma, seems to solve a problem of melanoma resistance to standard treatment. Suchcationic dendritic peptides: have some advantages (1) low toxicity fornormal cells, (2) rapid penetration through cell membranes, (3) the interaction with specific surface and intracellular targets in tumor cells, including, cell surface nucleolin (NCL). This chaperone protein is highly expressed in solid tumors and trigger ligand internalization, including cationic peptides. Nucleolin and nucleophosmin (NPM) control the most important cell functions - DNA transcription, RNA translation, ribosome biogenesis, chromatin remodeling, cell signaling, differentiation, invasion, angiogenesis and carcinogenesis.
Volume II Issue 6 - 2020

\author{
Anna A Lushnikova,' Galina B Smirnova,' \\ AleksandrV Kostarev, ${ }^{2}$ Anastasia $V$ Onyan,' \\ Anna A Rudakova,' Ksenia V Kozhikhova, ${ }^{3}$ \\ Sergej M Andreev ${ }^{3}$ \\ 'NN Blokhin Cancer Research Center, Russia \\ ${ }^{2}$ Boston Gene, Moscow Branch, Russia \\ ${ }^{3}$ Immunology Institute of FMBA, Russia
}

Correspondence: Anna A Lushnikova, Carcinogenesis Institute, N.N. Blokhin Cancer Research Center, II 5478 Kashirscoye Sh-24, Russia, Tel +74993235866,

Email lanI@yandex.ru

Received: November 17, 2020 | Published: November 27, 2020

However, NCL/NPM expression in normal tissues is significantly lower. Differential expression levels of NCL and NPM in tumor and normal cells allows to use these proteins as a targets for selective inhibition of tumor growth. Molecular mechanisms for $\mathrm{CP}$ selective cytotoxicity and specific antitumor effect are related with functional inhibition of NCL/NPM followed by nucleolar stress this idea was proven by in vivo experiments using melChermice subcutaneous xenografts.

Conclusion: Tumor growth suppression up to $85 \%$ was induced by two tested cationic peptides (CPs) in MelCher xenografts. High selective cytotoxicity of PCs tested for three cutaneous melanoma cell lines was also revealed invitro. Molecular docking results indicate high possibility for NCL/NPM interactions with CP molecule as cell targets and ligands with significant glide scores. Thus, CPs might consider as perspective anticancer agents.

Keywords: metastatic cutaneous melanoma (CM), cell lines, subcutaneous mice xenografts, cationic peptides, cell proliferation, selective inhibition

\section{Introduction}

A major challenge in the treatment of cutaneous melanoma is the development of metastases due to rapid tumor growth and late diagnostics. Finding a novel molecular targets and the design of targeted therapeutic approaches to improve the overall survival and quality of life of patients with melanoma is of great importance. Nucleolin, which is over expressed in cancer cells and tumorassociated blood vessels, have been implicated in various processes supporting tumorigenesis and angiogenesis. Additionally, its over expression has been demonstrated in a variety of human cancers as an unfavorable prognostic factor, associated with a high risk of relapse and low overall survival. Hence, nucleolin has emerged as a relevant target for therapeutic intervention in cancer malignancy. Nucleolin targeting has recently been shown to impaire the growth of primary tumors and liver metastasis in the orthotopicmouse models of pancreatic ductal adenocarcinoma by pseudopepetide N6L. Such nucleolin targeted strategy resulted in dual effect: inhibition of pancreatic adenocarcinoma progression and normalization of tumor vasculature.
Many synthetic polymers have been developed as drug delivery systems or therapeutic tools with high effectiveness and low toxicity for normal tissues. Some polymers are also used for early cancer diagnostics In vitro investigation of tumor cell lines obtained from various sources, allows to study tumor biology and mechanisms of the response to these anticancer agents.. This paper describes activities of some original cationic peptides (CPs), inducing selective apoptosis both in stable melanoma cell lines obtained from surgery biopsies, and their subcutaneous mice xenografts. Roles of NCL and NPM as expected cell targets and mechanism of induced by CPs tumor cell death are also discussed.

\section{Materials and methods}

The original stable cell lines melIS and melH were obtained from the lymph nodes of the female patients with metastatic cutaneous melanoma after 10 cell passages. In both cell lines BRAF V600E mutations were revealed by PCR followed by Sanger sequencing of amplified DNA. Other cell line MelCher (BRAF+) was obtained after 15 passages from the metastatic lymph node of the female patient 
diagnosed with back cutaneous melanoma. ${ }^{1}$ This line was used for subcutaneous MelCher xenograft generation in immunodeficient mice.

Normal human fibroblast line H1036 was used as a control .The cells were cultured in standard RPMI 1640 medium supplied with $10 \%$ fetal calf serum. Fifteen cationic peptides with different structure, molecular mass from 1090 to 2338 Dalton and molecular charge from $4+$ to $16+($ Table 1$)$ were tested by standard MTT cell proliferation assay(MTT viability test).

Table I Cationic peptides with antiproliferative activity for melanoma cells in vitro

\begin{tabular}{|c|c|c|}
\hline Peptide formula & $\begin{array}{l}\text { Molecular mass } \\
\text { (IDa)/charge }\end{array}$ & $\begin{array}{l}\text { Effective } \\
\text { concentration } \\
(\mu \mathrm{g} / \mathrm{ml})\end{array}$ \\
\hline $\mathrm{NC}-8 \mathrm{I} \mathrm{I} / \mathrm{R}_{8} \mathrm{~K}_{4} \mathrm{~K}_{2} \mathrm{KAC}-\mathrm{NH}_{2}$, & $2338 / 16+$ & $\geq 0.75$ \\
\hline $\mathrm{NC}-798 / \mathrm{K}_{8} \mathrm{~K}_{4} \mathrm{~K}_{2} \mathrm{KAC}-\mathrm{NH}_{2}$ & $2114 / 16+$ & $\geq 0.25$ \\
\hline $\mathrm{NC}-803 /(\mathrm{RRRKK})_{2} \mathrm{~K}_{3} \mathrm{AC}-\mathrm{NH}_{2}$, & $2026 / 14+$ & $\geq 0.50$ \\
\hline $\mathrm{NC}-780 /(\mathrm{K})_{4}(\mathrm{~K})_{2} \mathrm{KAC}-\mathrm{NH}_{2}$ & $1090 / 8+$ & $\geq 1.0$ \\
\hline AM2/Mir-KRPARPAR-NH ${ }_{2}$, & |391.6/4+ & $\geq 0.50$ \\
\hline $\mathrm{NC}-8 \mathrm{I0} / \mathrm{Palm}-\mathrm{C}(\mathrm{R})_{11}-\mathrm{NH}_{2}$ & $2207 / 12+$ & $\geq 0.25$ \\
\hline
\end{tabular}

Cationic peptides were synthesized by solid phase method by the strategy based on using of Fmoc-protective groups, diluted in sterile distilled water in concentrations from $0.25 \mu \mathrm{g} / \mathrm{ml}$ to $4.0 \mu \mathrm{g} / \mathrm{ml}$ for cell lines or $4.0 \mu \mathrm{g} / \mathrm{ml}$ insterile sodium chloride. $0.9 \%$ - forxenograft.

In vitro cytotoxicity was evaluated by standard MTT assays using 2-dayscell cultures in 96 well plates. Cell survival is reported as percentage of viable cells in the presence of CP (dilutions $0.25-4 \mu \mathrm{g} /$ $\mathrm{ml}$ ) versus untreated cells. Typically, cell survival values were obtained from at least three independent experiments and three determinations were performed for each sample. Standard deviations were calculated for the results obtained from all the experiments.

Immunohistochemical staining was performed on slides with fixed cell suspension incubated with anti-nucleolin NUC4E2 (Dia-m/ Abcam) monoclonal antibodies for $45 \mathrm{~min}$ at 370C. Secondary mouse antibodies were applied after blocking with Protein Block using EnVision kit (LabBiotech, Russia).

Mechanisms of cell death induced by the peptides were analyzed according standard protocols, using flow cytometry and Caspase 3 , Caspase 8, Caspase 9 (active) FITC Staining Kits, Abcam, Russia, c.N.ab65613, ab65614, ab65615, correspondingly.

In vivo experiments were performed using MelCher xenografts obtained from corresponding cell culture suspension $(5 \times 106$ cells for injection subcutaneously per immunodeficient. $(n=5)$ and control $(n=5)$ mice. Two CPs $-\mathrm{NC} 783$ and AM-2 were used for injections.

Molecular interactions were confirmed by simulation binding. The binding of small molecules to cellular proteins is an essential point of the protein function mechanisms. Chemical compounds or drugs that bind to active sites of the proteins allow modifying or inhibiting their functions. Identification of CPs as a potential ligands to target NCL and NPM proteins has been done by molecular docking using Maestro program described previously. ${ }^{2,3}$

\section{Results}

Nucleolin clusters on external side of cell membranes in spite of absence of hydrophobic domain associated with the membrane. The clustering is related with intracellular actin cytoskeleton. Cell surface NCL level is dependent on proliferating potential of nonmalignant and tumor cells. In normal cells surface NCL is self-cleaved followed by degradation. Overexpression of NCL mRNA in high proliferating tumor cells results in rapid nucleolin synthesis and translocationto cell surface as a receptor for variousligands. ${ }^{4}$ Overexpression of NCL and NPM proteins in all melanoma lines was confirmed by western blotting (is not shown). Chaperone proteins NCL and NPM with high expression level in tumor cells of different origin are considered as a perspective targets for different therapeutic and other macromolecules, bacteria and viruses, including many classes of cell-penetrating agents. ${ }^{5}$ These agents require cell surface nucleolin for translocation across cell membranes and activation signaling pathways. Intracellular NCL and NPM are the first docking proteins for transport and folding of various ligands. These proteins regulate the most important functions for cell viability. Nucleolin has a wide range of activities as a shuttle between cell nucleus, cytoplasm and membrane. Cell surface NCL expression in different tumors is much more abundant than in non-malignant tissues. ${ }^{6,7}$

According the results of MTT- assay, the highest inhibitory activity was shown for CPs with dendritic structure. Hyperbranched structure of dendrimers enhance CP bioactivity due to high molecular stability and resistance for proteolysis. We have firstly revealed that these R/K-rich peptides have selective cytotoxicity for cutaneous melanoma cells caused by tumor cell apoptosis without clear toxicity for morphologically normal cells (Figure 1).

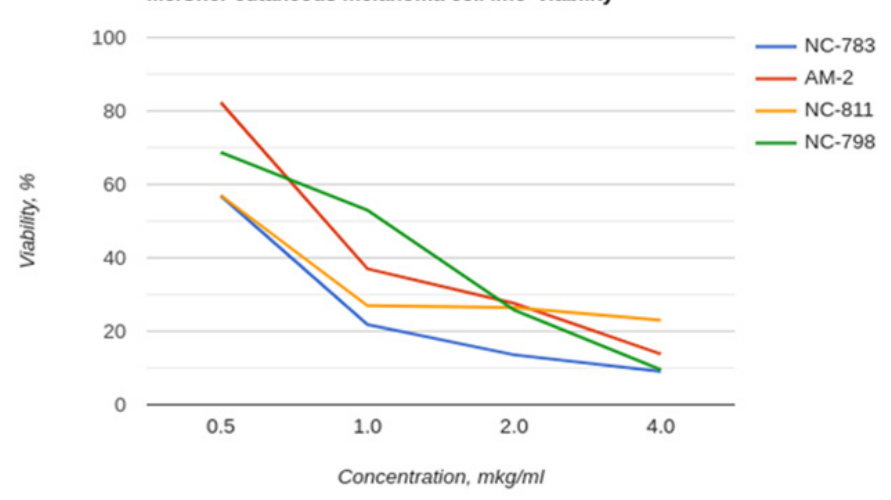

Figure I MTT assay results, obtained on melanoma MelCher line after $72 \mathrm{~h}$ cell cultivation including $48 \mathrm{~h}$ incubation with corresponding PC. Viability of morphologically normal human fibroblasts (control line HI036) was $100 \pm 4.3 \%$ for these PC concentrations.

Hyperbranched structure of dendrimers enhance CP bioactivity due to high molecular stability and resistance for proteolysis. Caspase 3 is activated by initiator caspases 8,9 or 10 and it specifically activates the endonuclease CAD in proliferative cells. The activation of caspases leads to reorganization of the cytoskeleton and cell disintegration, stimulating apoptosis. We assume that CPs bind to their target (receptor) - membrane NCL and activate initiatory caspases $8,9,10$, then caspases 3,6 with subsequent inactivation of cellular functions. The destruction of cell compartments during apoptosis and fragmentation of chromosomal DNA result in inactivation of the CAD enzyme complex (carbamoyl-phosphate synthetase 2, aspartate transcarbamoylase, and dihydroorotase) combined with inhibitor of CAD - ICAD, as well as a blockage of CAD release 
by caspase 3, DNA fragmentation and degradation of nucleosomes. Inactivation of enzymes, involved in DNA repair, includes poly(ADPribose)- polymerases, or PARP, which were first described as caspase substrates. The PARP family is involved in the repair of DNA damage and chromatin remodeling due to poly-ADP-ribosylation of histones.
During apoptosis, caspase 3 inactivates PARP-1 and DNA repair. The destruction of DNA is also enhanced due to inactivation of topoisomerase II by caspases. Activation of caspases 3, 8,9 has been revealed in melanoma cells after cultivation in presence CP (Figure 2).

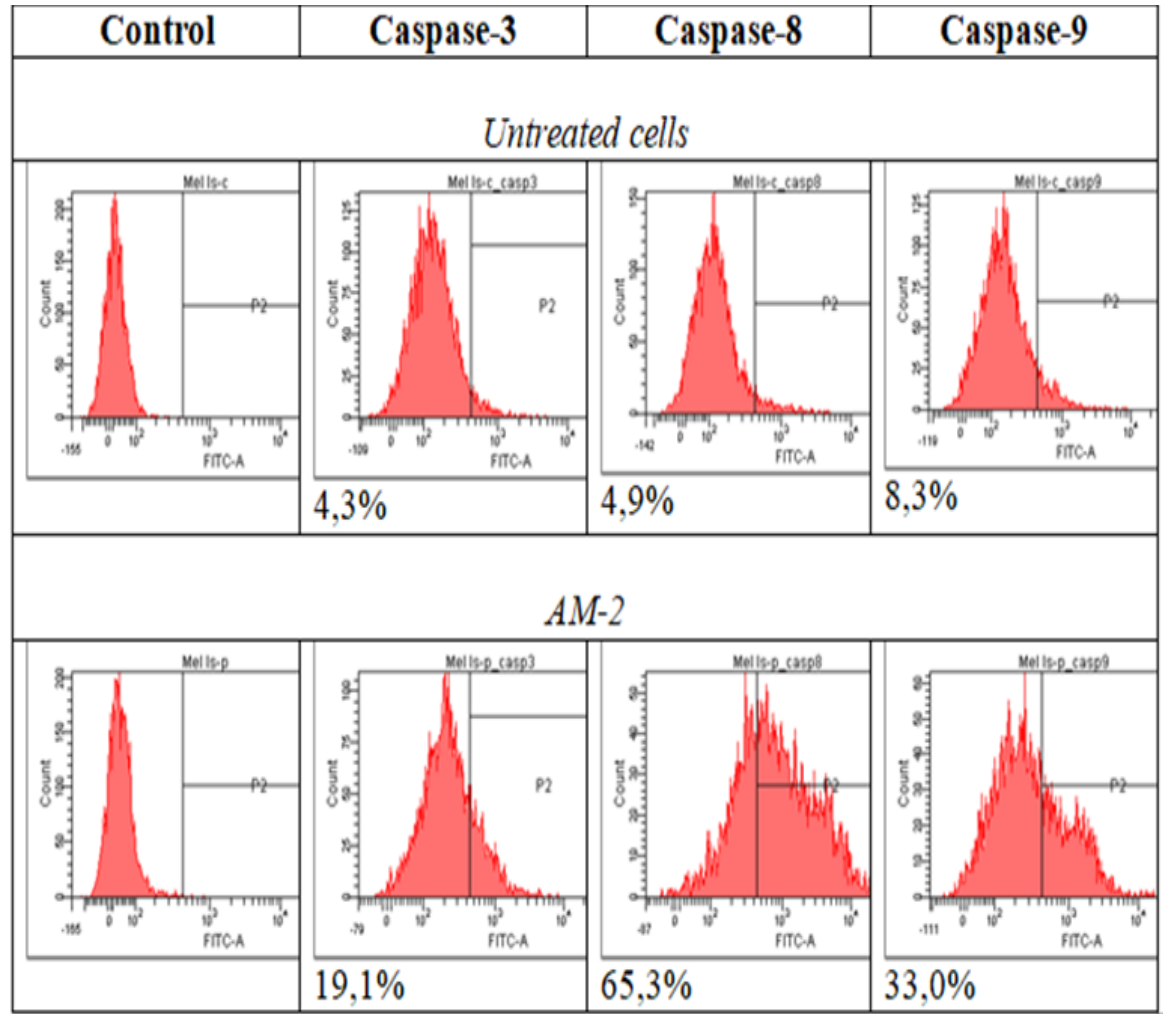

Figure 2 Caspase activation induced by CP AM-2 $(I \mu g / m l)$ in Mel Is cell line.

We have firstly revealed that these $\mathrm{R} / \mathrm{K}$-rich peptides have selective cytotoxicity for melanoma cells caused by tumor cell apoptosis without clear toxicity for morphologically normal cells. Thus, cytotoxicity of the cationic peptides is associated with ability to induce apoptosis. We have revealed that a number of $\operatorname{Arg}(\mathrm{R}) / \mathrm{Lys}(\mathrm{K})$ rich CPs are potential ligands for membrane NCL and form clasters, binding with active sites of NCL dimmer (RNA binding domains, RBD) followed by reduction of its functional activity and degradation of tumor cells by apoptosis and related processes. We have evaluated that key amino acids that are significant for NCL-ligand binding are Asp, Glu (q-, pH7.0), Arg, Lys, (q+, pH7.0), Thr (q0, pH7.0), located in NCL active center, including RB domains, and forming hydrogen bonds with some $\mathrm{NH} 2$ groups in peptide molecule. The results were confirmed by molecular docking (Figure $3 \& 4$ ).

Analysis of molecular binding interactions has revealed at least 10 amino acids in NCL/NPM active centres which form hydrogen bounds with cationic peptides in $49-100 \%$ interactions followed by inhibition of chaperon activity and apoptosis of tumor cells. Study of the pair binding of nucleolin (target protein) with CPs as a ligands has shown that the most peptide fragments bind to the active center of NCL dimmer with glide score values $>(-) 6.50$. Moreover, the hydrogen bonds between amino acids in NCL molecule and peptide ligand are formed not randomly. Really, an average frequencies of the hydrogen bonds for five amino acid positions in NCL active center exceeded $70 \%$, and aspartic acid, position 87 was associated with all the peptides under study. Peptide molecules are enriched with $\mathrm{R} / \mathrm{K}$ amino acid residues which also interact with the active center of nucleolin.

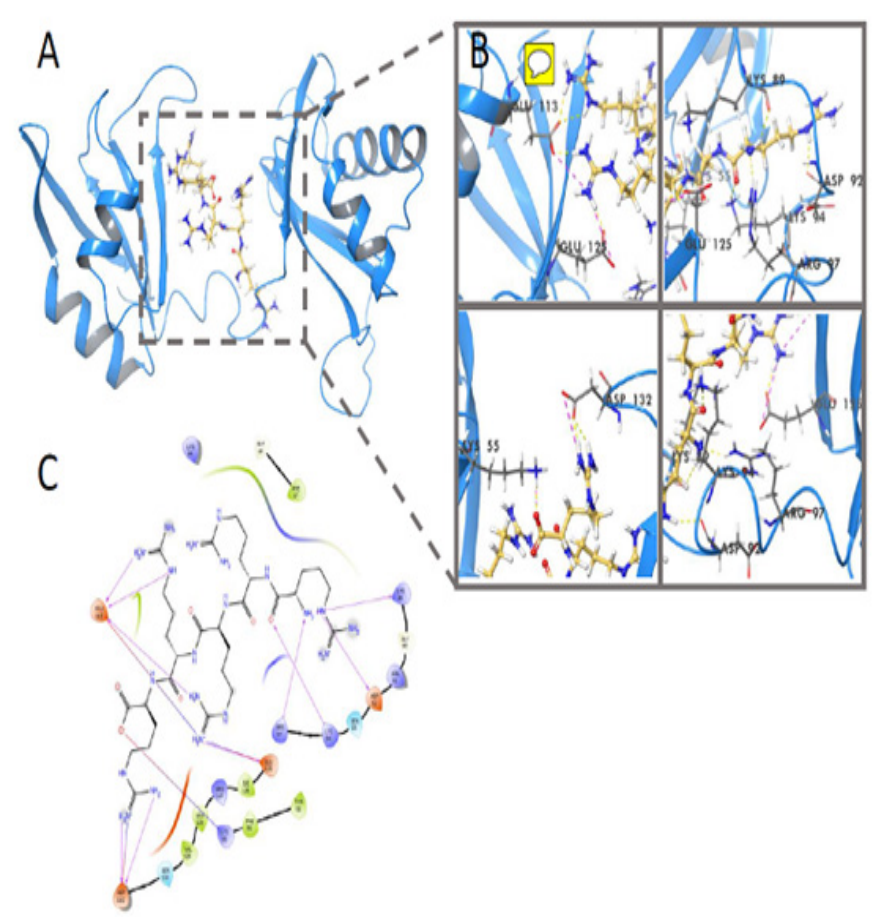

Figure 3 Molecular docking NCL-NC810, glide score $=-7.549 \mathrm{kcal} / \mathrm{mol}$. Yellow - hydrogen bounds between peptide(ligand) and nucleolin active centre (target).

Citation: Lushnikova AA, Smirnova GB, Kostarev AV, et al. Perspective approach for cutaneous melanoma treatment by cationic peptides.J Cancer Prev Curr Res. 2020; I (6):I39-|42. DOI: 10.15406/jcpcr.2020.II.00442 


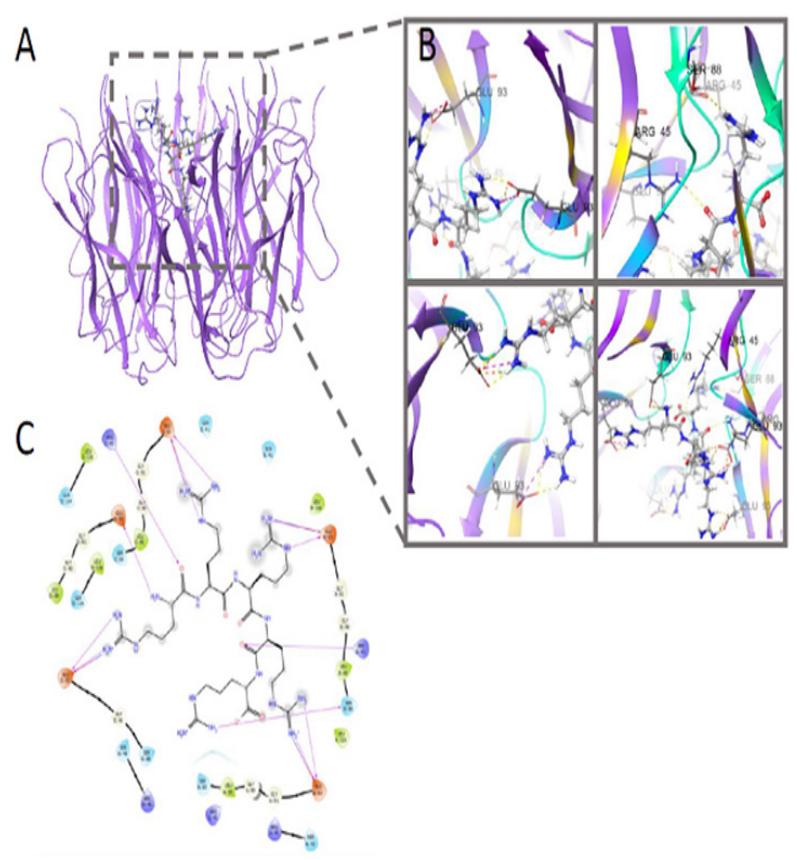

Figure 4 Molecular docking NPM-NC8I0, glide score=- $10.786 \mathrm{kcal} / \mathrm{mol}$.

Yellow - hydrogen bounds between peptid (ligand) and nucleophosmin active centre (target).

An analysis of the pair binding of nucleolin (target protein) with CPs as a ligands has shown that the most peptide fragments bind to the active center of NCL dimer with glide score values $>(-) 6.50$. Moreover, the hydrogen bonds between amino acids in NCL molecule and peptide ligand are formed not randomly. Really, an average frequencies ofthe hydrogen bonds for five amino acid positions in NCL active center exceeded $70 \%$, and aspartic acid, position 87 was associated with all (100\%) peptides under study. Peptide molecules are enriched with $\mathrm{R} / \mathrm{K}$ amino acid residues which also interact with the active center of nucleolin.

Preliminary in vivo experiments using MelCher melanoma xenografts indicate significant suppression of subcutaneous tumor growth (Figure 5). It confirms the data obtained in vitro and allow considering these peptides as a perspective anticancer agents.

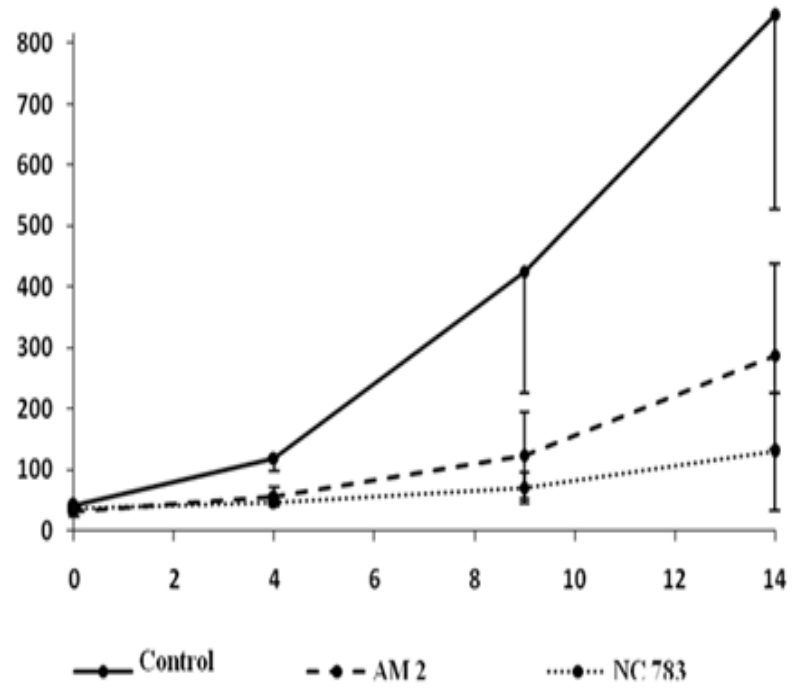

\section{Conclusion}

Induction of melanoma cell apoptosis by cationic peptides enriched with $\mathrm{R} / \mathrm{K}$ amino acid residues, is demonstrated in this study. Moreover, inhibitory effect of the peptides on tumor growth and angiogenesis in melanoma xenografts is established. These data suggests that these peptides represent a promising anticancer drug candidates warranting further investigation. The mechanisms of their antitumor activity is discussed.

\section{Submission declaration}

This work has not been published previously it is not under consideration for publication elsewhere. Its publication is approved by all authors. If accepted, it will not be published elsewhere in the same form, in English or in any other language, including electronically without the written consent of the copyright holder.

\section{Acknowledgments}

The authors are appropriate to Dr Lidia F Morozova for generous gift of melanoma cell lines.

\section{Conflicts of interest}

The authors have no competing interests to declare.

\section{Funding}

This project is supported by State Scientific Program of Russian Health Ministry.

\section{References}

1. Kandarakov OF, Kalashnikova MV, Vartanian AA, et al. Homogenous and heterogenous 3D melanoma models in vitro. Mol Biol. 2015;49(6):9981001 .

2. Lushnikova AA, Andreev SM, Kozhikhova KV, et al. Cationic peptides as the inductors of selective apoptosis in tumor cells. Allergy. 2018;73(Suppl 105):488.

3. Lushnikova AA, Kostarev AV, Onyan AV, et al. Simulation binding between nucleolin and cationic peptides, inducing tumor cell apoptosis, by molecular docking. J Cancer Prev Curr Res. 2018;9(4):187-189.

4. Lushnikova AA, Ponkratova DA, Kostarev AV, et al. Nucleolin and nucleophosmin as expected targets for cationic peptides, inducing tumor cell apoptosis. Journal Bioinformatics and Genomics. 2018;3(8):1-8.

5. Weidle UH, Maisel DA, Klostermann S, et al. Intracellular proteins displayed on the surface of tumor cells as targets for therapeutic intervention with antibody-related agents. Cancer Genomics \& Proteomics. 2011;8(2):49-54.

6. Romano S, Fonseca N, Simões S, et al. Nucleolin-based targeting strategies for cancer therapy: from targeted drug delivery to cytotoxic ligands. Drug Discov Today. 2019;24(10):1985-2001.

7. Jia W, Yao Z, Zhao J, et al. New perspectives of physiological and pathological functions of nucleolin (NCL). Life Sci. 2017;186:1-10.

Figure 5 MelCher melanoma xenograft. 